\title{
MUSE observations of the counter-rotating nuclear ring in NGC $7742^{\star}$
}

\author{
Thomas P. K. Martinsson ${ }^{1,2,3}$, Marc Sarzi ${ }^{4}$, Johan H. Knapen ${ }^{2,3}$, Lodovico Coccato ${ }^{5}$, Jesús Falcón-Barroso ${ }^{2,3}$, \\ Bruce G. Elmegreen ${ }^{6}$, and Tim de Zeeuw ${ }^{5,7,8}$ \\ ${ }^{1}$ Centro de Astrobiología (CAB, CSIC-INTA), Ctra de Torrejón a Ajalvir, 28850 Torrejón de Ardoz, Madrid, Spain \\ e-mail: tmartinsson@cab.inta-csic.es \\ ${ }^{2}$ Instituto de Astrofísica de Canarias, 38205 La Laguna, Tenerife, Spain \\ ${ }^{3}$ Departamento de Astrofísica, Universidad de La Laguna, 38206 La Laguna, Tenerife, Spain \\ ${ }^{4}$ Centre for Astrophysics Research, University of Hertfordshire, Hatfield, Hertfordshire AL1 9AB, UK \\ ${ }^{5}$ European Southern Observatory, Karl-Schwarzschild-Str. 2, 85748 Garching, Germany \\ ${ }^{6}$ IBM T. J. Watson Research Center, 1101 Kitchawan Road, Yorktown Heights, NY 10598, USA \\ 7 Sterrewacht Leiden, Leiden University, Postbus 9513, 2300 RA Leiden, The Netherlands \\ ${ }^{8}$ Max Planck Institut für extraterrestrische Physik, Giessenbachstrasse, 85748 Garching, Germany
}

Received 7 April 2017 / Accepted 22 November 2017

\begin{abstract}
Aims. We present results from MUSE observations of the nearly face-on disk galaxy NGC 7742. This galaxy hosts a spectacular nuclear ring of enhanced star formation, which is unusual in that it is hosted by a non-barred galaxy, and because this star formation is most likely fuelled by externally accreted gas that counter-rotates with respect to its main stellar body.

Methods. We used the MUSE data to derive the star-formation history (SFH) and accurately measure the stellar and ionized-gas kinematics of NGC 7742 in its nuclear, bulge, ring, and disk regions.

Results. We have mapped the previously known gas counter-rotation well outside the ring region and deduce the presence of a slightly warped inner disk, which is inclined at approximately $6^{\circ}$ compared to the outer disk. The gas-disk inclination is well constrained from the kinematics; the derived inclination $13.7^{\circ} \pm 0.4^{\circ}$ agrees well with that derived from photometry and from what one expects using the inverse Tully-Fisher relation. We find a prolonged SFH in the ring with stellar populations as old as 2-3 Gyr and an indication that the star formation triggered by the minor merger event was delayed in the disk compared to the ring. There are two separate stellar components: an old population that counter-rotates with the gas, and a young one, concentrated to the ring, that co-rotates with the gas. We recover the kinematics of the old stars from a two-component fit, and show that combining the old and young stellar populations results in the erroneous average velocity of nearly zero found from a one-component fit.

Conclusions. The spatial resolution and field of view of MUSE allow us to establish the kinematics and SFH of the nuclear ring in NGC 7742. We show further evidence that this ring has its origin in a minor merger event, possibly 2-3 Gyr ago.
\end{abstract}

Key words. techniques: imaging spectroscopy - galaxies: structure - galaxies: kinematics and dynamics galaxies: individual: NGC 7742

\section{Introduction}

Nuclear rings are important structures for the evolution of disk galaxies. The rings are prime tracers of disk galaxy dynamics and give information on the underlying dynamical structure of galaxies. They are also indicators of angular momentum transport, a key factor in secular evolution.

The advantages of studying rings are that they are common, bright, and easily observable. Star-forming nuclear rings occur in $\sim 20 \%$ of disk galaxies and are found in SO-Sd galaxies with a peak in Sab-Sb galaxies (Knapen 2005; Comerón et al. 2010). They are the site of intense star formation, with rates $\sim 100$ times what is observed in galactic disks (e.g. Hsieh et al. 2012).

Nuclear rings are generally thought to form as gas accumulates at special orbital resonances while it is channeled

\footnotetext{
* Data used for the flux and kinematic maps (Figs. 1 and 3-5) are only available at the CDS via anonymous ftp to cdsarc.u-strasbg.fr (130.79.128.5) or via http://cdsarc.u-strasbg.fr/viz-bin/qcat?]/A+A/612/A66
}

towards the central regions of a galaxy by bars (Combes \& Gerin 1985; Heller \& Shlosman 1994; Knapen et al. 1995). Their formation, evolution, and demise are therefore central to the secular growth of the central stellar-mass concentrations of galaxies and may be responsible for the creation of so-called pseudo-bulges (Kormendy \& Kennicutt 2004). They are also relevant to the fueling of central supermassive black holes, as they essentially halt such fueling, and more generally to the connection between AGN and central star-formation activity (Kauffmann et al. 2003).

In the order of $20 \%$ of the star-forming nuclear rings occur in disk galaxies where a stellar bar is absent (Comerón et al. 2010). The source NGC 7742 belongs to this minority group. This source is a nearly face-on SA(r)b galaxy with a morphology dominated by its nuclear ring. The ring has a radius of $9^{\prime \prime}$ (Comerón et al. 2010), or $\sim 1.0 \mathrm{kpc}$ assuming the distance to the galaxy $D=22 \mathrm{Mpc}$ (Mazzuca et al. 2006). The size of the galaxy disk is $R_{25}=52^{\prime \prime}$ (or $\sim 6.0 \mathrm{kpc}$ ). There is no evidence for current interactions with other galaxies, although it may form a pair with NGC 7743, which 

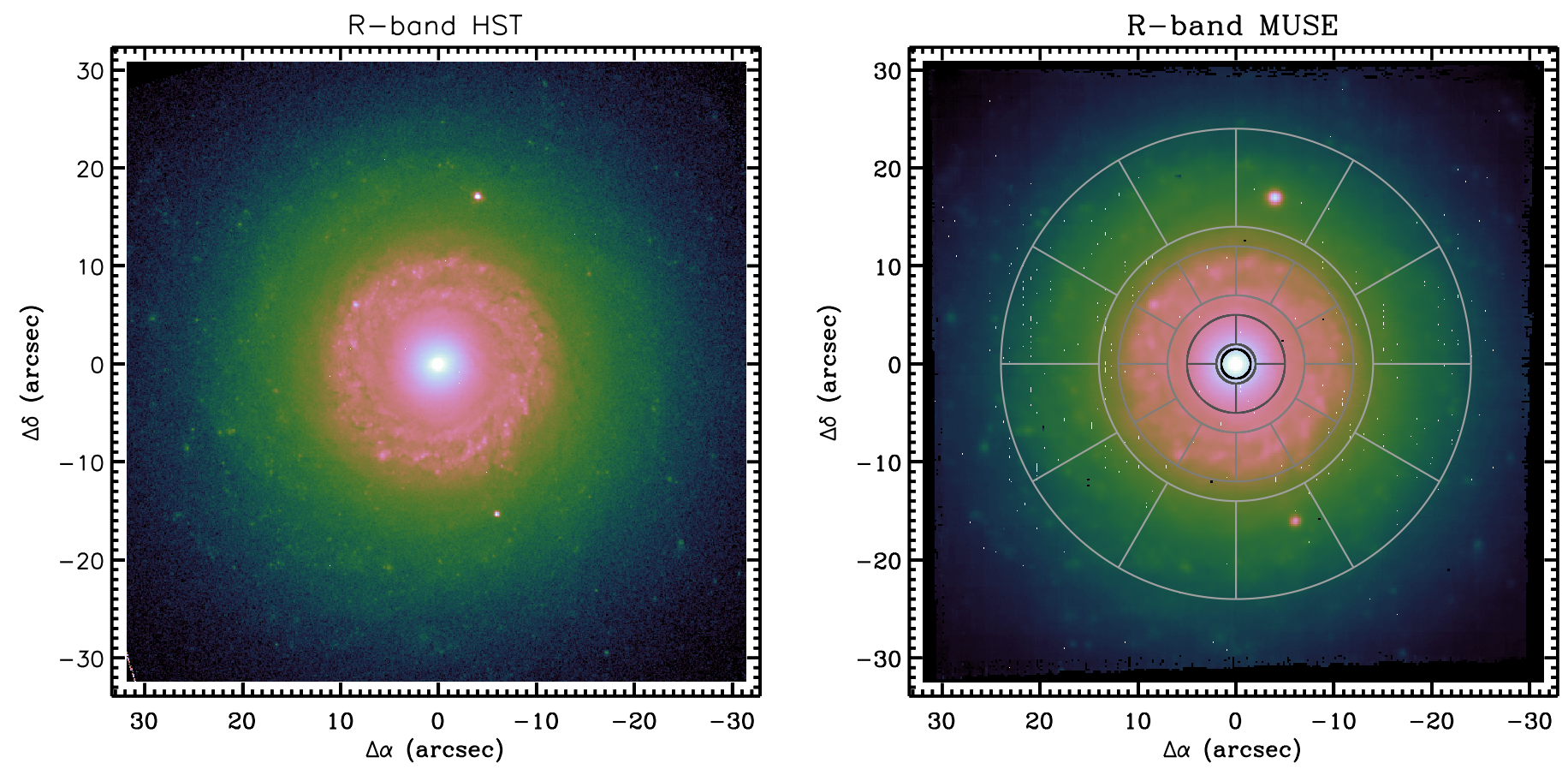

Fig. 1. HST vs. MUSE. Left: Hubble Space Telescope R-band image of NGC 7742. North is up and east is to the left. The $60^{\prime \prime}$ side is $\sim 7 \mathrm{kpc}$ wide in physical scale, assuming a distance to NGC 7742 of $22 \mathrm{Mpc}$. Right: MUSE reconstructed $R$-band image of NGC 7742, with the locations of 29 apertures overplotted. These apertures were used to extract the high-S/N spectra that provided the optimal stellar templates at the basis of our stellar kinematic and gas measurements. The apertures were designed to sample the nuclear, bulge, ring, and disk regions, allowing us to also investigate the SFH across these regions. The two foreground stars were masked out during the extraction of the aperture spectra.

is $320 \mathrm{kpc}$ away and within $50 \mathrm{~km} \mathrm{~s}^{-1}$ in radial velocity (Mazzuca et al. 2006).

Most non-barred nuclear-ring galaxies show evidence for an alternative mechanism to obtain non-axisymmetry in the gravitational potential, such as strong spiral patterns, weak oval distortions, or disturbances due to a companion galaxy (Buta \& Combes 1996). Minor merger events have also been suggested to trigger the formation of nuclear rings in unbarred galaxies (e.g. Knapen et al. 2004), and NGC 7742 is an ideal case for testing such ideas given that a counter-rotation between the gas in the ring and the stars in the bulge (de Zeeuw et al. 2002) reveal an external origin for this nuclear ring. The ring was interpreted as a result of a minor merger already by Mazzuca et al. (2006) and Sil'chenko \& Moiseev (2006). That interpretation seems adequate, since a major merger would completely destroy the disk while anything less than a minor merger would have difficulties resulting in counter-rotation in the central region.

Investigation of galaxies with counter-rotating components, such as NGC 7742, is important for understanding galaxy evolution, as it gives us information on previous merger and accretion events and the role of these events in the evolution of galaxies. For example, mergers have been shown as a mechanism for the formation of S0 galaxies (e.g. Bekki 1998), which could explain why counter-rotation is more common in these morphological types (Corsini \& Bertola 1998; Corsini 2014; Bassett et al. 2017). Again, NGC 7742 is a special galaxy here, in that only about $10 \%$ of spiral galaxies have counter-rotating disks (Pizzella et al. 2004).

The early SAURON integral-field maps of de Zeeuw et al. (2002) first presented evidence for an external origin of the nuclear ring of NGC 7742. Later SAURON measurements of Falcón-Barroso et al. (2006) served to trace the transition in the gas properties from the nucleus into the ring when they were combined with DensePak data in Mazzuca et al. (2006). Sarzi et al. (2007) placed constraints on the star-formation history of the nuclear ring of NGC 7742 using long-slit measurements and, like for other objects in their study, found that this ring formed most likely over a prolonged period of time (a few $100 \mathrm{Myr}$ ) characterized by episodic bursts of star-formation activity (see also Allard et al. 2006).

Mazzuca et al. (2006) used SAURON and DensePak data and only covered the central $1.5 \mathrm{kpc}$ of NGC 7742. Thanks to the relatively large field of view (FoV) of MUSE, we go out further in the disk, reaching $R>3 \mathrm{kpc}$. Together with the high spatial resolution and extended wavelength range, the MUSE data allow us to separate the stellar-population properties of the bulge, ring, and disk regions and to robustly separate the kinematics of the main stellar body of NGC 7742 from that of the newly acquired gas and the stars that formed from it.

After a short description of the observing strategy, data reduction, and data analysis in Sect. 2, we present our results in Sect. 3. In Sect. 4, we summarize these results.

\section{Observations, reduction, and analysis}

\subsection{Observing strategy}

Observations of NGC 7742 were carried out with the Multi-Unit Spectroscopic Explorer (MUSE; Bacon et al. 2010) on June 22, 2014, during the instrument science verification in the context of Programme 60.A-9301 (PI Sarzi). MUSE is a second generation integral field spectrograph that consists of 24 identical integral field units (IFUs), and operates on the Unit Telescope 4 (UT4) of the Very Large Telescope (VLT) in Paranal, Chile.

We used MUSE in the wide-field spatial mode and the nominal wavelength mode. The wide-field mode covers a 

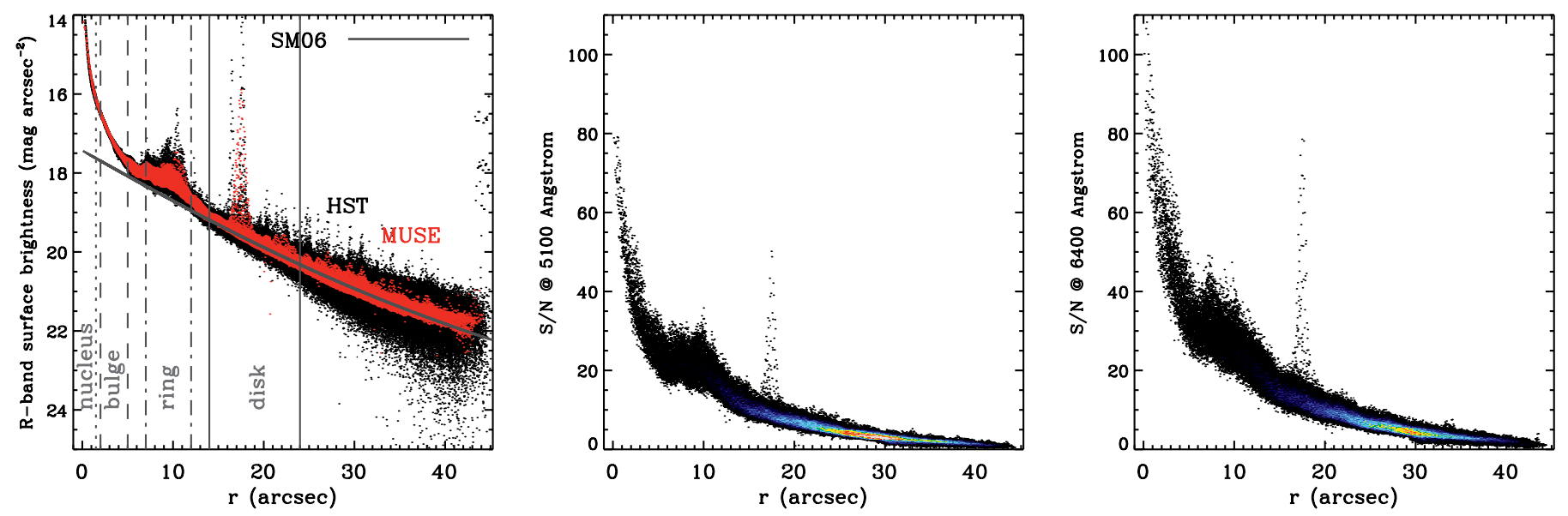

Fig. 2. Surface brightness and $\mathrm{S} / \mathrm{N}$ in individual spaxels as a function of radius. Left: $R$-band surface brightness as a function of radius, from HST (black dots) and MUSE (red dots) data. The grey line indicates the two-disk model of Sil'chenko \& Moiseev (2006) rescaled to $R$-band, highlighting the regions dominated by the disk surface brightness. The radial extent of the apertures shown in Fig. 1 are also indicated. Middle: $\mathrm{S} / \mathrm{N}$ as a function of radius and per spectral resolution element, at $5100 \AA$. Right: same as middle, but now at $6400 \AA$. The colours in the middle and right panels indicate the density of individual spaxel measurements (redder colours means higher density).

$60^{\prime \prime} \times 60^{\prime \prime}$ FoV with a spatial sampling of $0.2 \times 0.2$. The spectroscopic nominal mode covers a 4800-9300 A wavelength range with a dispersion of $1.25 \AA$ pixel $^{-1}$ and a nominal resolving power that ranges from 1770 (at $4800 \AA$ ) to 3590 (at $9300 \AA$ ).

The observations were organized in two exposures of $1800 \mathrm{~s}$, offset by $1^{\prime \prime}$ in declination and rotated by $90^{\circ}$. One offset exposure of $300 \mathrm{~s}$ was taken in between for the evaluation of the sky background. As part of the standard MUSE calibration plan, a set of bias, internal flat fields, sky flat fields, and arc-lamp exposures were taken, and a spectro-photometric standard star was observed for photometric calibration.

\subsection{Data reduction}

We used the MUSE pipeline (v1.6; Weilbacher 2015) within the Reflex environment (Freudling et al. 2013) to reduce the data. For each on-sky exposure, we used the flat fields that were taken within $12 \mathrm{~h}$ from the scientific exposure, and that were closer in temperature. In our case, flats closer in temperature to the observations were also closer in time. Large-scale illumination variations were corrected via sky flat fields, obtained during twilight. The white dwarf star GD 153 was observed at twilight with the same observational set-up of NGC 7742, and it was used to correct for the instrument response and telluric absorption.

The contributions of the night sky emission lines and continuum were evaluated from the dedicated offset exposure. The pipeline constructed a model of the sky spectrum and subtracted it from the two scientific exposures, after scaling it for exposure time and after convolving it for the instrumental line-spread function for each slice and IFU.

Figure 1(left panel) shows the HST $R$-band image, which can be compared to the reconstructed MUSE $R$-band image (right panel). The comparison illustrates the quality of the data. As can be seen, the seeing was very good ( $F W H M \sim 0$.' $^{\prime}$, measured from the two foreground stars) during the observations.

Figure 2 further illustrates the quality of the MUSE data by comparing the surface brightness of the HST and the reconstructed MUSE images as well as showing the run of signalto-noise ratio $(\mathrm{S} / \mathrm{N})$ in each individual MUSE spectrum (that is prior to any spatial binning) as a function of radius.

\subsection{Data analysis}

In order to extract the stellar and gas kinematics and measure the gas flux distribution in NGC 7742, we first proceeded to spatially bin our MUSE cube using the Voronoi tessellation method of Cappellari \& Copin (2003) and a formal target $S / N=30$ in each bin. Such a $\mathrm{S} / \mathrm{N}$ target is sufficient to accurately measure the stellar kinematics over the relatively long wavelength range afforded by the MUSE spectra ${ }^{1}$, which in turn ensures robust emissionline measurements. For this purpose, we used the penalized pixel fitting (pPXF) code of Cappellari \& Emsellem (2004) to extract the stellar kinematics and subsequently measure the emissionline flux and kinematics using the GandALF code of Sarzi et al. (2006).

When running the pPXF and GandALF procedures on the Voronoi-binned spectra we used a predefined set of optimal stellar templates that were based on the full MILES single-age stellar-population models of Falcón-Barroso et al. (2011). More specifically, these optimal templates correspond to the best combination of the MILES models that were needed during the pPXF and GandALF fit to 29 high-S/N aperture spectra covering the central $24^{\prime \prime}$ of NGC 7742, where we obtained an optimal template for each of the 29 individual apertures. As shown in Fig. 1, these apertures were designed to sample the nuclear, bulge, ring, and disk regions of NGC 7742, where variations of the stellar-population properties are more likely to occur. In fact, the analysis of such aperture spectra also allowed us to estimate the star-formation history in these four regions (Sect. 3.5).

It should be noted that the naming of the different regions is only indicative. The "ring" apertures were based on the extent of

\footnotetext{
1 We settled on a $S / N=30$ target for our spatial binning after measuring the stellar kinematics on the MUSE data and observing how the formal pPXF errors on the velocity and velocity dispersion fare as a function of $\mathrm{S} / \mathrm{N}$. This experiment showed that, within our chosen wavelength range and with an appropriate set of pre-defined optimal templates (such as we obtain from our apertures), by a $S / N=30$ we reach a precision of $\sim 10 \mathrm{~km} \mathrm{~s}^{-1}$ and $\sim 15 \mathrm{~km} \mathrm{~s}^{-1}$ in the stellar velocity and velocity dispersion, respectively. This is also broadly consistent with that found from simulations of SAMI data by Fogarty et al. (2015), which is not surprising given that they also have a fairly long wavelength range that includes several stellar absorption features.
} 

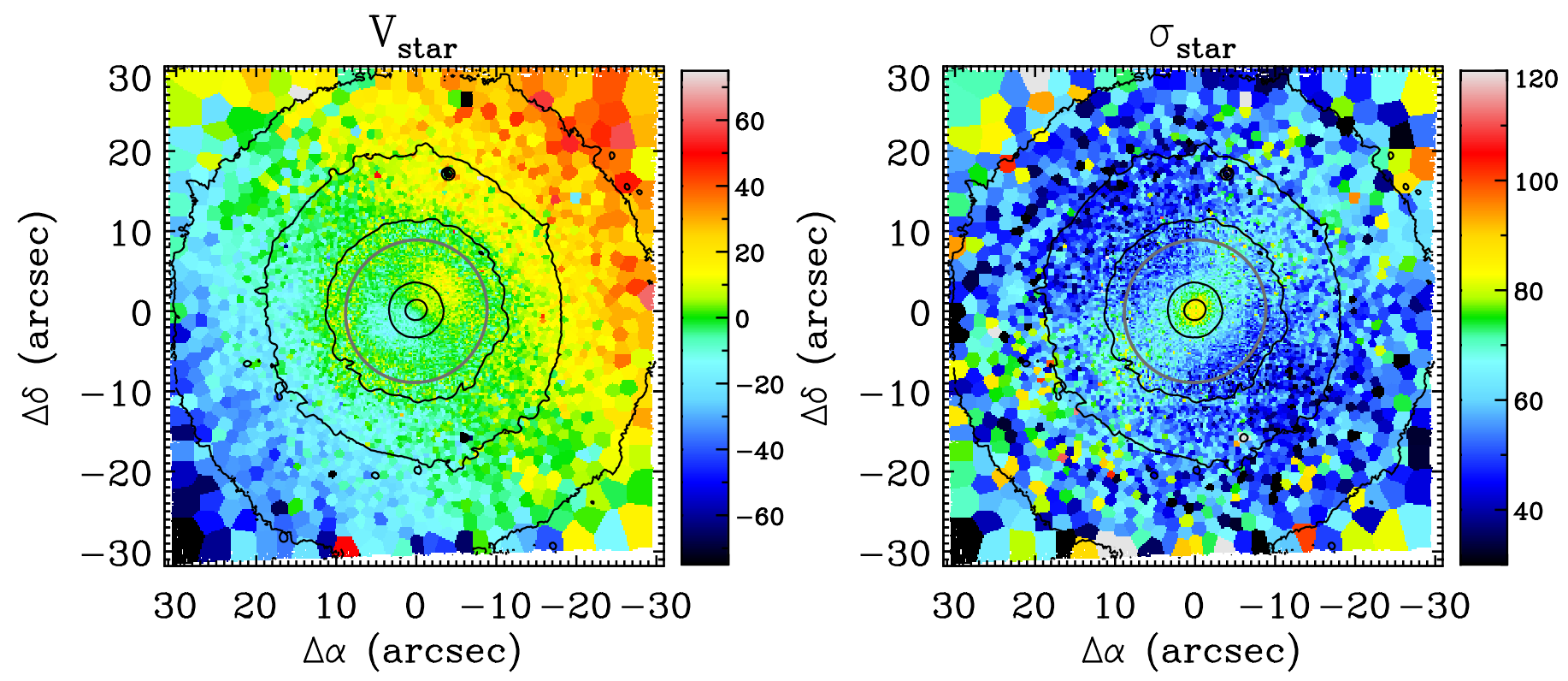

Fig. 3. Stellar kinematics. Left: stellar velocity field. Right: stellar velocity dispersion map. The black contours indicate surface brightness levels in the Hubble F675W image (Fig. 1), in intervals of half a magnitude. The grey ellipse in each figure at $R=9^{\prime \prime}$ indicates the location of the ring as defined by Comerón et al. (2010).

the ring in the $\mathrm{H} \alpha$ image. The "disk" apertures were extracted from the same number of apertures out to a radius where we only include spectra with sufficient $\mathrm{S} / \mathrm{N}$. The "bulge" region is taken to be inside the ring and subdivided into four regions, plus a central "nuclear" region. However, the two-disk model of Sil'chenko \& Moiseev (2006) in Fig. 2 shows that what we call disk apertures are indeed dominated by the disk light, whereas the regions inside the ring see a substantial contribution from the bulge component.

\section{Results}

\subsection{Stellar kinematics}

Figure 3 shows the stellar velocity field and the stellar velocity dispersion map as obtained from our pPXF fits. These maps provide a clearer and more complete view of the stellar kinematics of NGC 7742 as first mapped by Falcón-Barroso et al. (2006), and also clearly show how the previously reported counterrotation between stars and gas extends well beyond the ring region (see gas maps in Fig. 5).

The MUSE data also bring out much more clearly the kinematic presence of the newly formed stars in the ring, and possibly also in the disk regions. As these new stars rotate in an opposite direction to that of the bulge stars, our pPXF measurements for the relative velocity estimates decrease to near-zero values along the kinematic major axis, where we also observe a similarly artificial increase in the stellar velocity dispersion. The pPXF measurements shown in Fig. 3 are indeed based on the assumption that a single velocity distribution would apply to all stars observed along the light-of-sight, which would then lead pPXF to estimate an average, nearly zero velocity in the presence of two counter-rotating stellar components, and to be similarly biased towards larger values for the velocity dispersion (Bertola et al. 1996; Krajnović et al. 2011). In order to show how the kinematics of these components can be separated, in Sect. 3.4 we use priors on the stellar-population properties for the main and newly born stellar population of NGC 7742.
We confirm a rather low stellar velocity dispersion in the centre $\left(\sigma_{*, 0}<90 \mathrm{~km} \mathrm{~s}^{-1}\right)$ as reported by Sil'chenko \& Moiseev (2006); for being of an early type, this galaxy is of rather low mass with only a minor inner mass concentration. The photometric disk decomposition in Sil'chenko \& Moiseev (2006) also results in only a small and modest bulge component.

\subsection{Gas kinematics}

In Fig. 4, we plot the ionized-gas ([NII] and $\mathrm{H} \alpha$ ) flux maps. These maps show that there is plenty of gas in this galaxy, with a concentration in the star-forming ring. The $\mathrm{H} \alpha$ flux, in particular, is clearly stronger where star formation is taking place. From these maps, more than 100 stellar clusters can be detected, with a particularly interesting one being located inside the ring, just $4^{\prime \prime}$ west of the centre seen most clearly in the $\mathrm{H} \alpha$ map. This stellar cluster proves that the star formation is ongoing also well inside the ring.

Figure 5 shows the ionized-gas velocity field and velocity dispersion map (produced for both [NII], where all forbidden lines were tied together, and $\mathrm{H} \alpha$, which show very similar kinematics; only the [NII] velocity field and velocity dispersion maps have been represented in Fig. 5). The velocity field displays rather regular rotation over the full $\mathrm{FoV}$, although one can note that it sometimes deviates significantly from that of a disk of gas in circular and coplanar orbits. There is a peak in the rotational velocity at about $6^{\prime \prime}$ from the centre, but as we see in Sect. 3.3 this is likely a projection effect from a warped inner disk.

The gas velocity dispersion map shows a dip in the dispersion at the location of the ring, which is likely due to the higher starformation activity and the associated requirement for gas to be in a dynamically cold state. Interestingly, a lower gas velocity dispersion can also be seen in the bulge stellar cluster mentioned above.

In the very centre, inside $2^{\prime \prime}$, there is a possible decoupled disk with a different position angle than the outer disk. This 

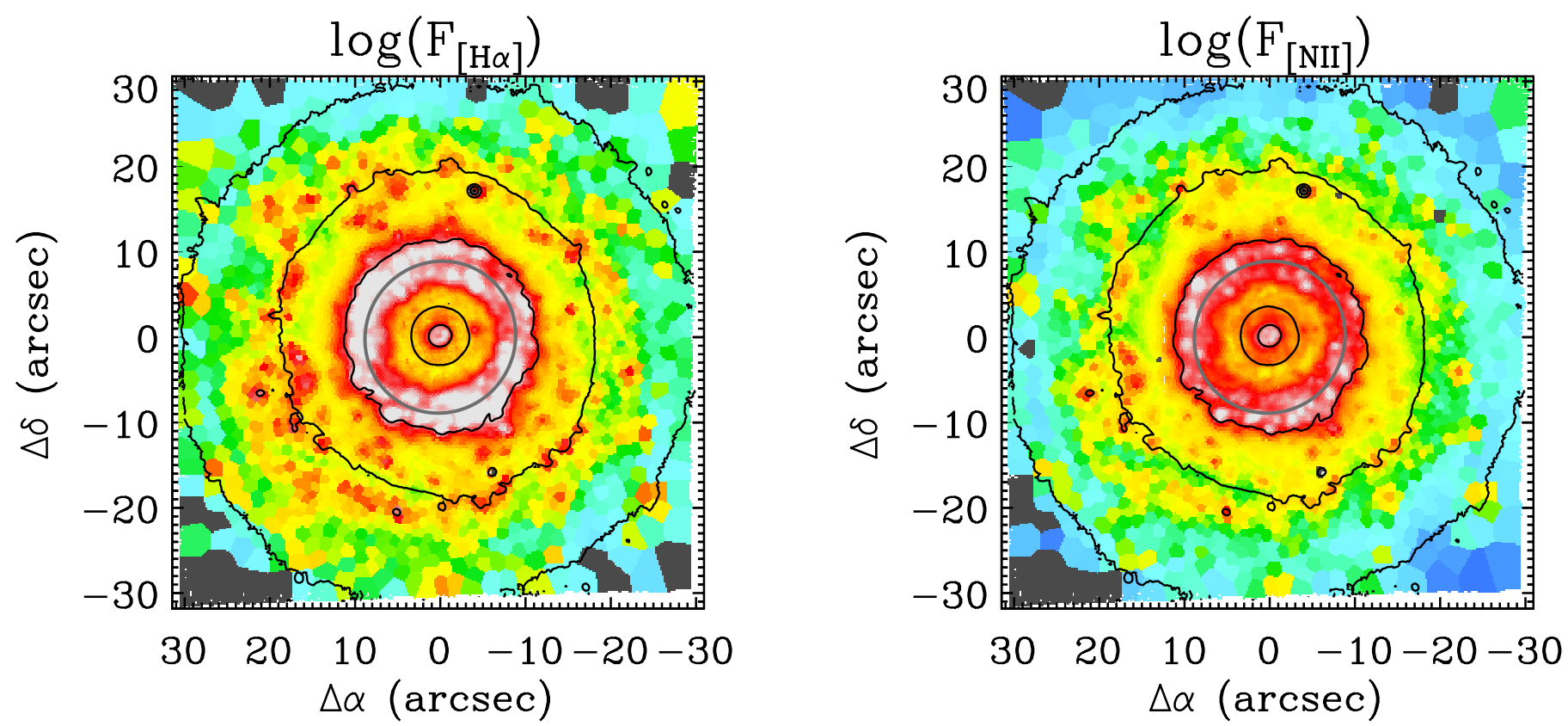

Fig. 4. Ionized-gas emission flux. Left: $\mathrm{H} \alpha$ flux map. Right: [NII] flux map. Redder colours indicate a higher flux, with white (seen in the ring region) representing the highest flux. Contours are the same as in Fig. 3. One can note the stellar cluster inside the ring, $4^{\prime \prime}$ to the west (right) of the centre, most clearly seen in the $\mathrm{H} \alpha$ map, and also seen as a dip in the velocity dispersion in Fig. 5.
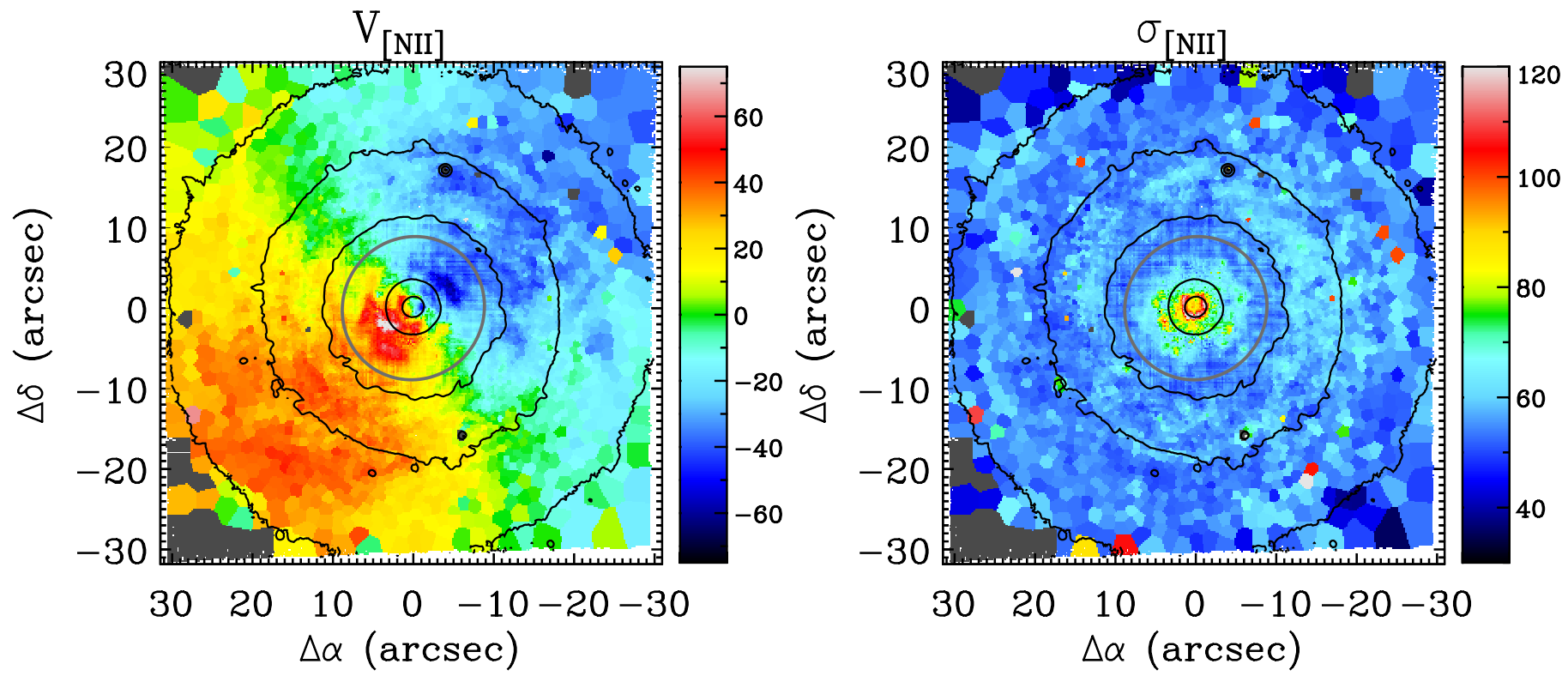

Fig. 5. Ionized-gas kinematics. Left: velocity field traced by the [NII] forbidden lines. Right: [NII] gas velocity dispersion. Contours are the same as in Fig. 3.

seems also associated with an inner "ring" of higher gas dispersion (located approximately at the most inner contour). However, we note that this may also be an AGN-related feature.

\subsection{A possible inner warp}

On the major axis of the gas velocity fields (Fig. 5), we note that the line-of-sight velocity is higher at about $R=6^{\prime \prime}$, then decreases when going out to larger radii before becoming nearly constant. A rapid rise of the rotation curve, followed by a dip before reaching its flat part, is often seen in massive disk galaxies with a major inner mass concentration (e.g. Sofue et al. 1999).
However, for NGC 7742, which has a modest rotational velocity of $\sim 150 \mathrm{~km} \mathrm{~s}^{-1}$ (see below) and does not appear to have a massive bulge (Sect. 3.1), we do not expect such a large amplitude difference. A possible explanation could be that we see a projection effect: a small warp in this nearly face-on galaxy will give large relative differences in the derived velocity.

To investigate this further, we performed a tilted-ring analysis using ROTCUR within the Groningen Image Processing SYstem (GIPSY) software package (van der Hulst et al. 1992; Vogelaar \& Terlouw 2001). In principle, all ring parameters can be fit simultaneously. In practice, however, there are degeneracies affecting the fit, in particular a significant covariance between the inclination $(i)$ of the ring and its rotational velocity 


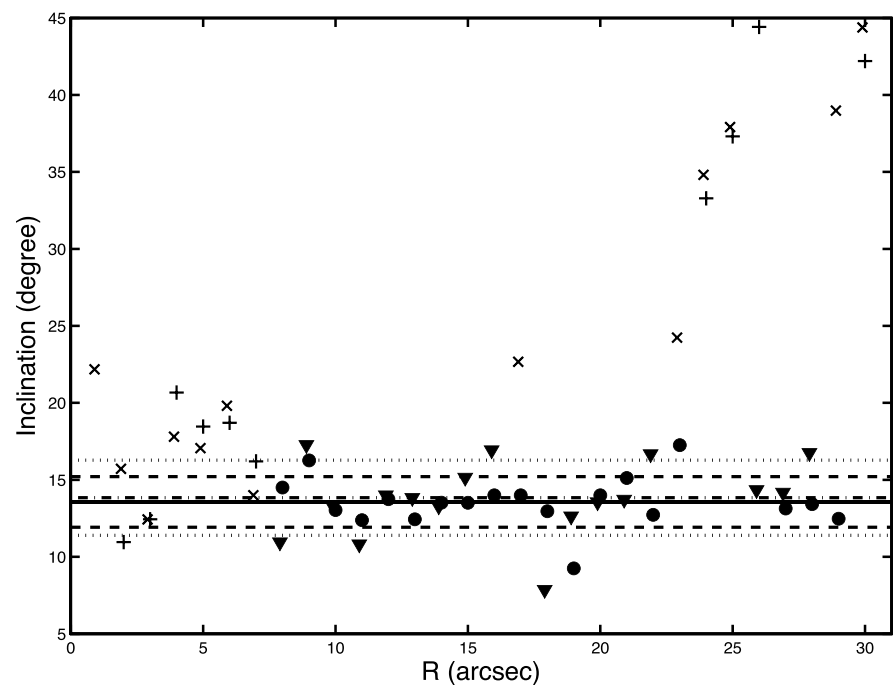

Fig. 6. Derived disk inclination of the $[\mathrm{NII}]$ and $\mathrm{H} \alpha$ gas disks. Circles and triangles show derived inclinations in $1^{\prime \prime}$ wide rings for the [NII] and $\mathrm{H} \alpha$ gas, respectively. Measurements that have been excluded are indicated with crosses $([\mathrm{NII}])$ and pluses $(\mathrm{H} \alpha)$. The solid and dashed lines show the calculated mean and standard deviation, respectively, for the [NII] gas. The dashed-dotted and dotted lines show the calculated mean and standard deviation, respectively, for the $\mathrm{H} \alpha$ gas.

$\left(V_{\text {rot }}\right)$ for galaxies seen nearly face-on. This degeneracy may yield large inclination errors for galaxies with $i \lesssim 40^{\circ}$ (Begeman 1989). However, Andersen \& Bershady (2013) showed that highquality optical IFU kinematic data can yield accurate and precise kinematic inclinations for a non-warped disk down to about $15^{\circ}$ when modelling the entire velocity field as a single, inclined disk.

Here we used traditional ring-fitting, fitting the various parameters [centre, systemic velocity $\left(V_{\text {sys }}\right)$, position angle (PA), $i$, and $\left.V_{\text {rot }}\right]$ in five steps, similar to the method described in Martinsson et al. (2013). Due to the degeneracy mentioned above, we did not expect to derive a useful kinematic disk inclination, but instead rely on the inclination calculated from photometry or inferred from the inverse Tully-Fisher relation, which is generally more precise in this inclination regime. However, after having derived $V_{\text {sys }}$ and $\mathrm{PA}^{2}$, the inclination measurements in the rings give consistent results, with relatively small formal errors.

Figure 6 shows the disk inclination of the [NII] and $\mathrm{H} \alpha$ gas disks derived from our ROTCUR fits in $1^{\prime \prime}$ wide rings. With the exception of the inner disk and some outliers in the outer disk, we find consistent inclination values in the rings for both [NII] and $\mathrm{H} \alpha$. For the [NII] and $\mathrm{H} \alpha$ disks we derive an average inclination and formal error in the mean of $13.6^{\circ} \pm 0.4^{\circ}$ and $13.8^{\circ} \pm 0.6^{\circ}$, respectively, with a standard deviation of $1.7^{\circ}$ for $[\mathrm{NII}]$ and $2.4^{\circ}$ for $\mathrm{H} \alpha$. The inclinations were derived after excluding all inner measurements ( $R<8^{\prime \prime}$; likely affected by the warp), and after an iterative three-sigma clipping to remove outliers. Combining the results from the two tracers, the derived gas-disk inclination (mean and error in mean) is $i_{\text {kin }}=13.7^{\circ} \pm 0.4^{\circ}$. The derived inclination agrees well with the value of $14^{\circ}$ found by Muñoz-Mateos et al. (2015) using Spitzer photometry, and also with $i_{\mathrm{TF}}=15^{\circ} \pm 1^{\circ}$ found from inverting the Tully-Fisher relation of Verheijen (2001; calculated as in Martinsson et al. 2013).

\footnotetext{
2 We find a gas-disk PA $=140 \pm 4^{\circ}$ for both tracers, excluding the inner
} measurements $\left(R<8^{\prime \prime}\right)$.

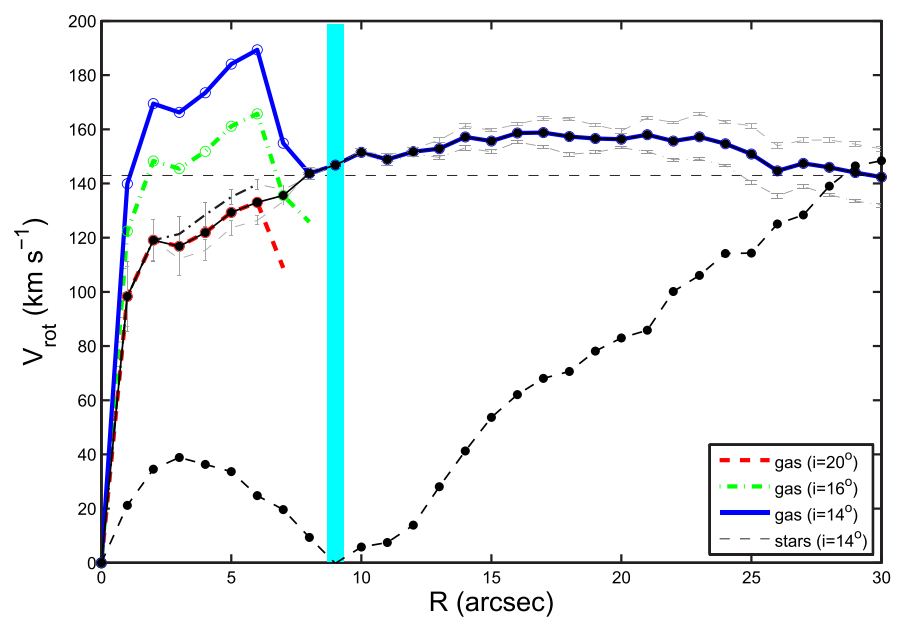

Fig. 7. Gas and stellar rotation curves. The coloured lines show fitted gas (combined [NII] and $\mathrm{H} \alpha$ ) rotation curves assuming three different disk inclinations (solid blue: $i=14^{\circ}$; dash-dotted green: $i=16^{\circ}$; dashed red: $i=20^{\circ}$ ). The two thin grey lines with error bars indicate the individual $[\mathrm{NII}]$ and $\mathrm{H} \alpha$ rotation curves. The dashed black line shows the fitted stellar rotation curve $\left(i=14^{\circ}\right)$. The thin dashed black horizontal line indicates the rotation speed expected from the inverse Tully-Fisher

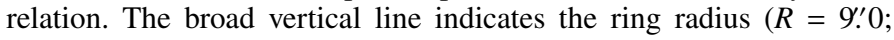
Comerón et al. 2010).

Considering a possible inner warp, in Fig. 7 we plot the derived rotation curve with a constant inclination of $14^{\circ}$ as a blue solid line. The derived rotation curve indeed shows a significant peak around $R=2-6^{\prime \prime}$. However, by setting the inclination of the tilted rings in the inner $6^{\prime \prime}$ to $20^{\circ}$, and $16^{\circ}$ in the intermediate region at 7 ", we obtain a rotation curve that is continuously rising; more what one would expect for a disk galaxy with a small inner mass concentration.

Sil'chenko \& Moiseev (2006) found that the innermost $(R<$ $\left.3^{\prime \prime}\right)$ gas disk is strongly warped, with an inner disk inclination $i_{\text {gas }}>35^{\circ}$. This could be a more strongly inclined circumnuclear disk (see also Sect. 3.2), separated from, but possibly affecting, the outer disk for which we find has only a modest warp of $6^{\circ}$.

\subsection{Stellar kinematic decomposition of the ring}

In Sect. 3.1, we noted the very low stellar velocities in the ring region, in particular along the kinematic major axis (Fig. 3). The stellar rotation curve (Fig. 7), derived in the same way as the gas rotation curve ${ }^{3}$ (Sect. 3.3), indeed behaves oddly with a broad dip at the radius of the ring. This is because thus far we have fitted the stellar kinematics assuming a single line-of-sight velocity distribution (LOSVD), whereas what we observe is the superposition of two distinct LOSVDs, one of the rotating old stars in the bulge and disk and the other of the counter-rotating young stars that formed mainly in the ring. When interpreting such a superposition as a single velocity distribution common to all stars along the line of sight, this leads to an LOSVD estimate centred near the average velocity from the two old and young stellar populations, which is close to zero in this case since these are counter-rotating, and also results in an increase in the measured velocity dispersion due to the shifts between the lines.

\footnotetext{
3 We find a stellar disk inclination of $12.1 \pm 4.9^{\circ}$, but here adopt $i=14^{\circ}$. The derived PA of the outer $\left(R>14^{\prime \prime}\right)$ stellar disk is $321^{\circ} \pm 1^{\circ}$, that is $\sim 180^{\circ}$ difference from the gas disk, as expected for aligned but counterrotating disks.
} 


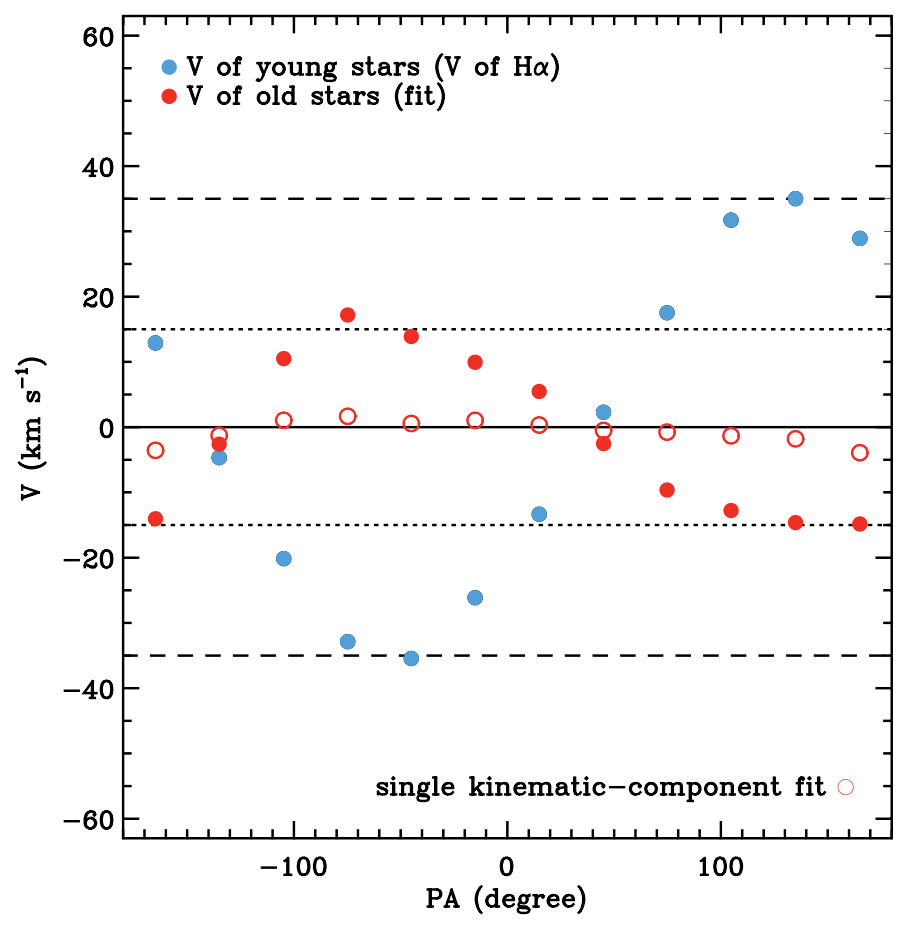

Fig. 8. Two-component fit to the stellar kinematics, plotted azimuthally along the ring. Empty red circles show the line-of-sight rotational velocity derived from a single kinematic component fit, while the filled red and blue circles show the rotation derived from the old stars and young stars (using $\mathrm{H} \alpha$ as a proxy), respectively.

One can separate the rotation of the old component from that of the newly formed stars in the ring by fitting two LOSVDs in the pPXF code, as done in Coccato et al. (2011) and more recently in Morelli et al. (2017). For this we used our 12 high-S/N ring apertures and the result of our stellar-population analysis (see Sect. 3.5) in order to create a "bulge" and a "ring" template by combining all single-age templates older and younger than 1 Gyr, respectively, according to their best fitting mass weights (see Fig. 9). Due to the low inclination of NGC 7742, and the consequent small velocity separation between the old and young stellar component, we found it necessary to impose to the young stars in the ring the same velocity as observed for the ionized $(\mathrm{H} \alpha)$ gas in order to converge to sensible results.

From the two-disk profile of Sil'chenko \& Moiseev (2006) indicated in Fig. 2, we estimate that the disk light contributes $\sim 67 \%$ of the $R$-band light in the ring region $\left(7^{\prime \prime}-12^{\prime \prime}\right)$, with the remaining $33 \%$ most likely belonging to recently formed stars contributing to the observed surface-brightness bump (the bulge light is not contributing much at this radius). This is consistent with both the result from the kinematic decomposition in this section and our stellar-population analysis in Sect. 3.5, where stars younger than 1 Gyr account to $\sim 35 \%$ and $\sim 32 \%$ of the light in the $R$-band, respectively.

Figure 8 shows the recovered velocity of the two components azimuthally along the ring, as well as the velocity derived from the single-component fit (which was used to derive the stellar rotation curve in Fig. 7). It is evident that an old stellar component and a young stellar component, counter-rotating in the same way as the ionized gas, can reproduce the observed single-component fit of nearly zero amplitude.

The superposition of the old and counter-rotating young components would also cause an overall broader line-of-sight velocity dispersion, in particular along the line of the nodes where co- and counter-rotating velocities would differ most. That
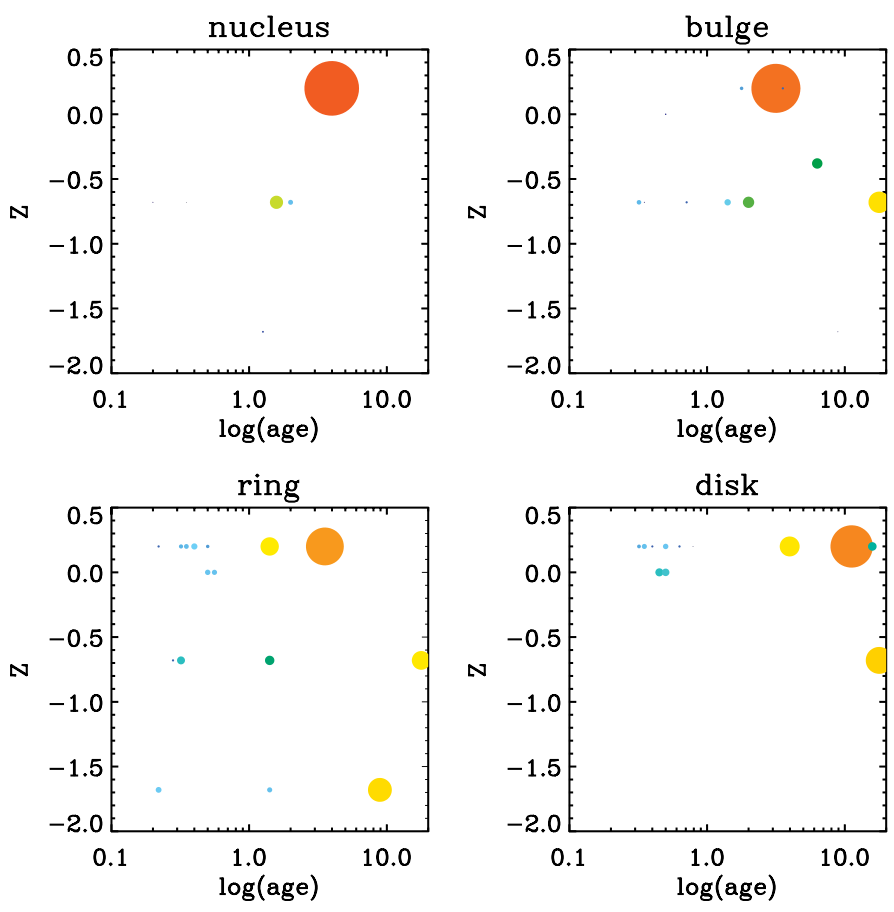

Fig. 9. Stellar-population properties (age and metallicity) in the nuclear, bulge, ring, and disk regions. Each dot shows the mass-weight assigned to the model used during the GandALF fit for the various apertures, where a larger and redder dot indicates a larger mass-weight.

is how the enhancement feature in the velocity dispersion maps can be interpreted, which is indeed aligned with the direction of the kinematics major axis in both the stellar and gas maps.

\subsection{Stellar populations and star-formation histories}

As described in Sect. 2.3, prior to extracting the stellar kinematics and carrying out our emission-line measurements in the Voronoi-binned spectra, we used pPXF and GandALF and the stellar-population model library of Falcón-Barroso et al. (2011) to fit 29 high-S/N spectra extracted within the apertures shown in Fig. 1. While this allows to use a more restricted set of optimal templates during the PPXF and GandALF fit to our Voronoi bins, thus speeding up this process, the results of our aperture fits also allow us to study the star-formation history $(\mathrm{SFH})$ in the nuclear, bulge, ring, and disk regions (but see Sect. 2.3 on the definition of these regions). The SFH can indeed be reconstructed using the weights assigned to each of the MILES single-age stellarpopulation models while fitting the aperture spectra, and more specifically when using GandALF, since during such a fit we use only multiplicative polynomials.

Figure 9 shows, as a function of their age, the mass-weight assigned to each of the MILES models that were used during the GandALF fit for the nuclear, bulge, ring, and disk apertures, without distinguishing the results from individual apertures covering the bulge, ring, and disk regions (that is, 4, 12, and 12, respectively). This was done on purpose, since we do not expect to be able to ascertain the presence of possible azimuthal gradients (for instance, in the ring regions the circular orbital period is just $20 \mathrm{Myr}$, well below the age of the youngest of our singleage templates). On the other hand, by effectively considering our different aperture spectra for the bulge, ring, and disk regions as independent probes of these regions we can instead convey in a conservative way the typical uncertainties in the templateweighting process that enters into the GandALF procedures. 

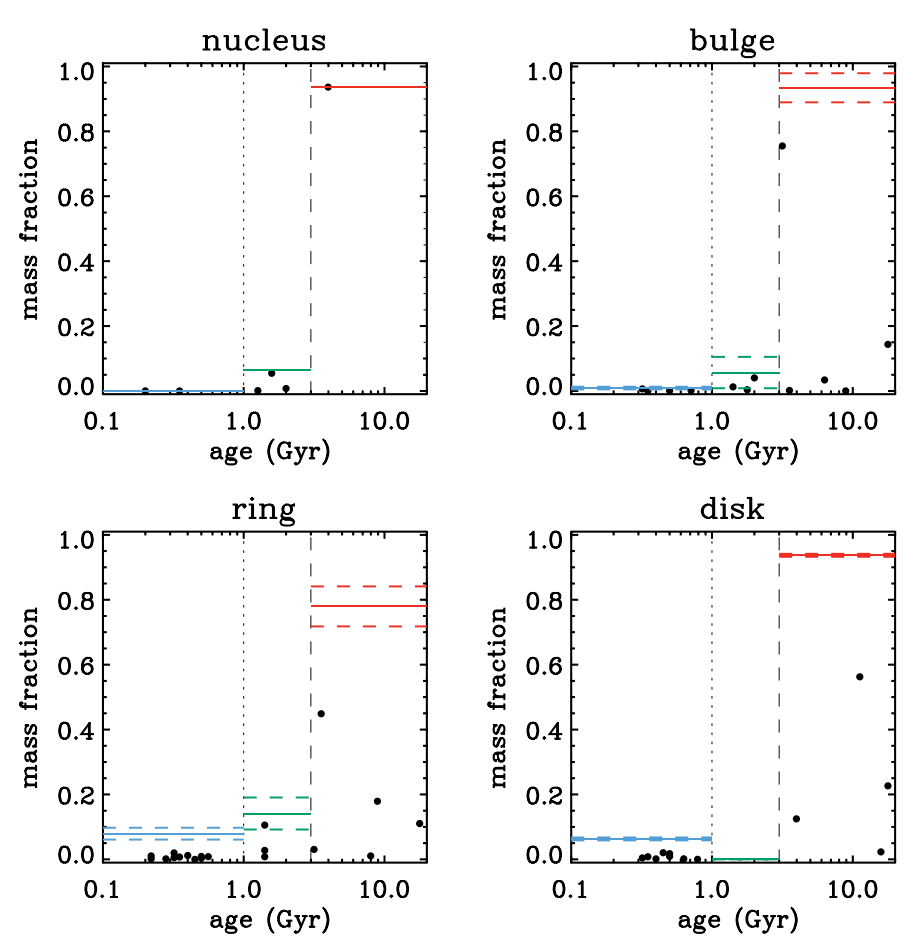

Fig. 10. Star-formation histories of the nucleus, bulge, ring, and disk regions. The black dots correspond to the coloured dots in Fig. 9. The locations of the horizontal blue, green, and red lines indicate the mass fraction of the young $(<1 \mathrm{Gyr})$, intermediate-old (1-3 Gyr), and old ( $>3 \mathrm{Gyr}$ ) stellar populations, respectively, calculated by adding the contributions from individual templates (black dots). The horizontal dashed lines indicate the formal errors in the mass fractions, deduce by treating each different azimuthal aperture spectrum as an independent probe of the bulge, ring, and disk regions. For the nucleus, errors should be taken to be similar to those reported for the bulge regions, which are similarly dominated by old stellar populations. We note that the disk measurements are very robust, whereas stochastic effects such as the presence of different numbers of HII regions contribute to the larger errors in the stellar-population analysis for the ring region.

To ease the reading of the SFH in NGC 7742, in Fig. 10 we group together the contribution of young (up to 1 Gyr old), intermediate (between 1 Gyr and 3 Gyr old) and old (above 3 Gyr) stellar populations. In this way, we see that young stars are predominantly found both in the ring and disk regions of NGC 7742, where they contribute, respectively, $8 \%$ and $6 \%$ to the total stellar mass, although some young stars (up to $1 \%$ ) also appear in the bulge. Intermediate-age populations are also present in the ring regions, where they add up to $14 \%$ of the mass, as well as in the bulge and nuclear regions (although to a lower extent). This is consistent with previous studies advocating a prolonged SFH in nuclear rings (e.g. Allard et al. 2006; Sarzi et al. 2007), and further suggests that stars formed in the ring may also migrate out of these structures. Interestingly, there is little or no evidence for intermediate-age stars in the disk regions, beyond the central ring. We speculate that this is an indication that whereas star formation in the ring was triggered soon after the merging event that NGC 7742 may have experienced 2-3 Gyr ago, gas in the outer regions took a longer time to settle down so that in the disk star formation only started in the past $1 \mathrm{Gyr}$.

\section{Summary}

We have presented results from MUSE observations of the nonbarred, nuclear-ring galaxy NGC 7742. The relatively large FoV of MUSE allowed us to map the stellar and gas kinematics much further out in the disk than what has been possible with previous data sets, while the high spatial resolution made it possible to derive the kinematics in the central parts of the galaxy.

From our tilted-ring analysis, we derived a gas-disk inclination of $i_{\text {kin }}=13.7^{\circ} \pm 0.4^{\circ}$ beyond the radius $R \approx 8^{\prime \prime}$. Within this radius, we identified an inner warp of the gas disk in the order of $6^{\circ}$, which due to projection causes a peak in the ionized-gas rotation curve around $R=6^{\prime \prime}$.

We confirmed the counter-rotation of gas and stars which presumably has a minor merger origin. The recently formed young stars, which are concentrated in (but not limited to) the ring, co-rotate with the gas and thus counter-rotate with respect to the old stars. A simple one-component fit to the stellar kinematics erroneously yields a low-amplitude stellar rotation curve, as highlighted when we combine the kinematics of the old stellar component with the counter-rotating young component assumed to move as the ionized gas to reproduce the observed one-component velocity in the ring.

We studied the $\mathrm{SFH}$, using high-S/N spectra extracted within large apertures in the nuclear, bulge, ring, and disk regions. Our results suggest a prolonged $\mathrm{SFH}$ in the nuclear ring over more than 1 Gyr. However, the lack of intermediate-age (1-3 Gyr) stars in the disk regions indicates that, although star formation in the ring was presumably triggered soon after the merging event that NGC 7742 experienced 2-3 Gyr ago, the gas in the outer regions took longer time to settle down and the star formation in the disk therefore started much later.

Acknowledgements. We want to thank the anonymous referee for the constructive comments and suggestions that helped improving this paper. We also thank Nate Bastian at ARI of Liverpool John Moores University for discussions and comments on the manuscript. T.P.K.M., J.H.K., and J.F-B. acknowledge financial support from the Spanish Ministry of Economy and Competitiveness (MINECO) under grant numbers AYA2013-41243-P, AYA2016-76219-P, and AYA2016-77237-C3-1-P. T.P.K.M. is also supported by grant ESP2015-68964 from the Ministerio de Economía y Competitividad of Spain. J.H.K. acknowledges financial support from the European Union's Horizon 2020 research and innovation programme under the Marie Skłodowska-Curie grant agreement No 721463 to the SUNDIAL ITN network. This research has made use of the NASA/IPAC Extragalactic Database (NED) which is operated by the Jet Propulsion Laboratory, California Institute of Technology, under contract with the National Aeronautics and Space Administration. Based on observations made with the NASA/ESA Hubble Space Telescope, and obtained from the Hubble Legacy Archive, which is a collaboration between the Space Telescope Science Institute (STScI/NASA), the European Space Agency (ST-ECF/ESAC/ESA) and the Canadian Astronomy Data Centre (CADC/NRC/CSA). Based on observations collected at the European Organisation for Astronomical Research in the Southern Hemisphere under ESO programme 60.A-9301(A).

\section{References}

Allard, E. L., Knapen, J. H., Peletier, R. F., \& Sarzi, M. 2006, MNRAS, 371, 1087

Andersen, D. R., \& Bershady, M. A. 2013, ApJ, 768, 41

Bacon, R., Accardo, M., Adjali, L., et al. 2010, in SPIE Conf. Ser., 7735 Bassett, R., Bekki, K., Cortese, L., Couch, W. 2017, MNRAS, 471, 1892 Begeman, K. G. 1989, A\&A, 223, 47

Bekki, K. 1998, ApJ, 502, L133

Bertola, F., Cinzano, P., Corsini, E. M., et al. 1996, ApJ, 458, L67

Buta, R., \& Combes, F. 1996, Fund. Cosmic Phys., 17, 95

Cappellari, M., \& Copin, Y. 2003, MNRAS, 342, 345

Cappellari, M., \& Emsellem, E. 2004, PASP, 116, 138

Coccato, L., Morelli, L., Corsini, E. M., et al. 2011, MNRAS, 412, L113

Combes, F., \& Gerin, M. 1985, A\&A, 150, 327

Comerón, S., Knapen, J. H., Beckman, J. E., et al. 2010, MNRAS, 402, 2462

Corsini, E. M. 2014, in Multi-Spin Galaxies, eds. E. Iodice, \& E. M. Corsini, ASP Conf. Ser., 486, 51

Corsini, E. M., \& Bertola, F. 1998, J. Korean Phys. Soc., 33, S574

de Zeeuw, P. T., Bureau, M., Emsellem, E., et al. 2002, MNRAS, 329, 513 
Falcón-Barroso, J., Bacon, R., Bureau, M., et al. 2006, MNRAS, 369, 529 Falcón-Barroso, J., Sánchez-Blázquez, P., Vazdekis, A., et al. 2011, A\&A, 532, A95

Fogarty, L. M. R., Scott, N., Owers, M. S., et al. 2015, MNRAS, 454, 2050

Freudling, W., Romaniello, M., Bramich, D. M., et al. 2013, A\&A, 559, A96

Heller, C. H., \& Shlosman, I. 1994, ApJ, 424, 84

Hsieh, P.-Y., Ho, P. T. P., Kohno, K., Hwang, C.-Y., Matsushita, S. 2012, ApJ, 747,90

Kauffmann, G., Heckman, T. M., Tremonti, C., et al. 2003, MNRAS, 346, 1055

Knapen, J. H. 2005, A\&A, 429, 141

Knapen, J. H., Beckman, J. E., Heller, C. H., Shlosman, I., de Jong, R. S. 1995, ApJ, 454, 623

Knapen, J. H., Whyte, L. F., de Blok, W. J. G., van der Hulst, J. M. 2004, A\&A, 423, 481

Kormendy, J., \& Kennicutt, Jr., R. C. 2004, ARA\&A, 42, 603

Krajnović, D., Emsellem, E., Cappellari, M., et al. 2011, MNRAS, 414, 2923

Martinsson, T. P. K., Verheijen, M. A. W., Westfall, K. B., et al. 2013, A\&A, 557, A130

Mazzuca, L. M., Sarzi, M., Knapen, J. H., Veilleux, S., Swaters, R. 2006, ApJ, 649, L79
Morelli, L., Pizzella, A., Coccato, L., et al. 2017, A\&A, 600, A76

Muñoz-Mateos, J. C., Sheth, K., Regan, M., et al. 2015, ApJS, 219, 3

Pizzella, A., Corsini, E. M., Vega Beltrán, J. C., Bertola, F. 2004, A\&A, 424, 447

Sarzi, M., Falcón-Barroso, J., Davies, R. L., et al. 2006, MNRAS, 366 , 1151

Sarzi, M., Allard, E. L., Knapen, J. H., Mazzuca, L. M. 2007, MNRAS, 380, 949

Sil'chenko, O. K., \& Moiseev, A. V. 2006, AJ, 131, 1336

Sofue, Y., Tutui, Y., Honma, M., et al. 1999, ApJ, 523, 136

van der Hulst, J. M., Terlouw, J. P., Begeman, K. G., Zwitser, W., Roelfsema, P. R. 1992, in Astronomical Data Analysis Software and Systems I, eds. D. M. Worrall, C. Biemesderfer, \& J. Barnes, ASP Conf. Ser., 25, 131

Verheijen, M. A. W. 2001, ApJ, 563, 694

Vogelaar, M. G. R., \& Terlouw, J. P. 2001, Astronomical Data Analysis Software and Systems X, eds. F. R. Harnden Jr., F. A. Primini, \& H. E. Payne, ASP Conf. Ser., 238, 358

Weilbacher, P. 2015, in Science Operations 2015: Science Data Management - An ESO/ESA Workshop, held 24-27 November 2015 at ESO Garching, available at https: //www . eso.org/sci/meetings/2015/Sciops2015.html 